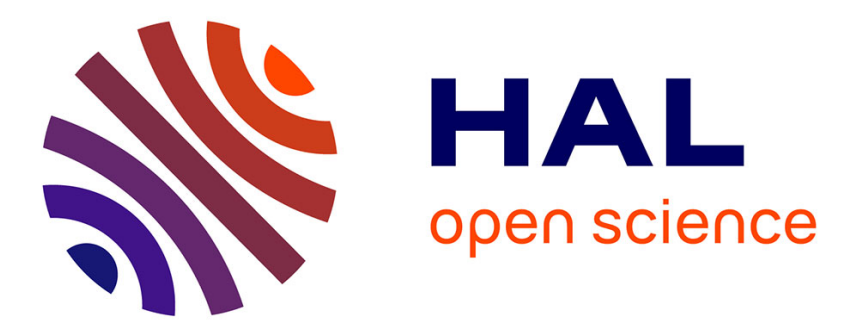

\title{
Syndrome du bébé secoué (SBS). Diagnostic et imagerie moderne
}

\author{
C. Adamsbaum, C. Rey-Salmon
}

\section{To cite this version:}

C. Adamsbaum, C. Rey-Salmon. Syndrome du bébé secoué (SBS). Diagnostic et imagerie moderne. Bulletin de l'Académie Nationale de Médecine, 2019, 203, pp.500 - 504. 10.1016/j.banm.2019.05.016 . hal-03488354

\section{HAL Id: hal-03488354 https://hal.science/hal-03488354}

Submitted on 21 Dec 2021

HAL is a multi-disciplinary open access archive for the deposit and dissemination of scientific research documents, whether they are published or not. The documents may come from teaching and research institutions in France or abroad, or from public or private research centers.
L'archive ouverte pluridisciplinaire HAL, est destinée au dépôt et à la diffusion de documents scientifiques de niveau recherche, publiés ou non, émanant des établissements d'enseignement et de recherche français ou étrangers, des laboratoires publics ou privés.

\section{다)(1) $(5$}

Distributed under a Creative Commons Attribution - NonCommercial| 4.0 International 


\title{
REVUE GÉNÉRALE
}

Reçu le 18/03/2019

Accepté le 6/05/2019

\section{Syndrome du bébé secoué (SBS). Diagnostic et imagerie moderne* Shaken baby syndrome. Diagnostic and modern imaging}

\author{
Catherine Adamsbaum ${ }^{1,2}$, Caroline Rey-Salmon ${ }^{3}$ \\ 1 Université Paris Sud, Faculté de Médecine, France \\ 2 AP-HP, CHU Bicêtre, Service de Radiologie Pédiatrique, 78 rue du Général Leclerc, \\ 94275 Le Kremlin Bicêtre Cedex, France \\ 3 AP-HP CHU Hôtel Dieu, Urgences médico-judiciaires, 1 place du Parvis Notre Dame 75004 Paris, France \\ Auteur correspondant : Catherine Adamsbaum \\ AP-HP, CHU Bicêtre, Service de Radiologie Pédiatrique, 78 rue du Général Leclerc, \\ 94275 Le Kremlin Bicêtre Cedex - France \\ E-mail : catherine.adamsbaum@aphp.fr
}

Les auteurs déclarent n'avoir aucun lien d'intérêt en relation avec le contenu de cet article.

* Séance du 7 mai 2019

\section{Résumé}

Le syndrome du bébé secoué (SBS) atteint surtout le nourrisson âgé de moins de 8 mois. Le mécanisme causal est une secousse ou une salve de secousses violentes, souvent répétée dans le temps. Les présentations cliniques sont variées, aiguës ou froides. Le diagnostic repose sur des arguments anamnestiques (histoire fluctuante ou incohérente), cliniques, ophtalmologiques et radiologiques. La recherche exhaustive d'autres lésions traumatiques concomitantes ou antérieures (ecchymoses, lésions endobuccales, fractures, etc.) est systématique. Le fait de secouer entraine des ruptures des veines-ponts, responsables d'un hématome sous dural caractéristique, multifocal, diffus et prédominant au vertex. Le scanner cérébral en urgence permet le diagnostic de SBS. L'IRM est utile pour l'analyse fine du parenchyme cérébral et des veines-ponts rompues. Le principal diagnostic différentiel de SBS dès lors que des ruptures de veines ponts sont présentes est le traumatisme accidentel à haute énergie cinétique. La datation des lésions concerne l'expertise médico-légale. La simple évocation du diagnostic de maltraitance doit conduire à l'hospitalisation immédiate de l'enfant dans un but de protection et pour la réalisation du bilan complet en imagerie : scanner cérébral complété si possible par une IRM, radiographies de haute définition de l'ensemble du squelette, échographie abdominale recommandée en France.

\begin{abstract}
Abusive head trauma (i.e. new name used for shaken baby syndrome) involves mainly infants under the age of 8 months. The causal mechanism is a violent shaking, often repeated over time. Clinical patterns are varied and may be acute or not. The diagnosis relies on a changing or inconsistent history, clinical, ophthalmological and radiological features. Other concurrent or previous traumatic injuries (bruises, endobuccal lesions, fractures, etc.) must be exhaustively looked for. Shaking is responsible for rupture of bridging veins that create a typical multifocal diffuse subdural hematoma which predominates at the vertex. The diagnosis of AHT relies on CT scan performed in emergency. MRI is useful for the fine analysis of the brain parenchyma, and the bridging veins. The main differential diagnosis of AHT as soon as a rupture of bridging veins is present, is high kinetic accidental trauma.
\end{abstract}


Dating of the injuries is a forensic issue. Any suspicion of abuse must lead to an immediate hospitalization to protect the child and perform a full imaging survey i.e. head CT with brain MRI as soon as possible, high definition full skeletal survey and abdominal ultrasound imaging.

Mots clés : Maltraitance des enfants, Syndrome du bébé secoué, Traumatismes cranioencéphaliques

Key words: Child Abuse, Shaken Baby Syndrome, Craniocerebral Trauma 


\section{Aspects épidémiologiques et cliniques}

Le syndrome dit du «bébé secoué » est la principale cause de mortalité et de morbidité au cours de la maltraitance du jeune enfant. En l'absence d'observatoire national, l'incidence en France est imprécise, estimée à plusieurs centaines (entre 14 et 56 cas pour 100000 enfants de moins de 1 an selon les études étrangères) [1]. L'incidence du SBS est sous-évaluée car elle n'intègre pas les enfants pour lesquels le diagnostic est manqué ni les décès attribués parfois rapidement à la mort subite du nourrisson. Il existe une nette prédominance masculine chez les victimes (environ $2 / 3$ de garçons) non expliquée à ce jour. Tous les milieux socioéconomiques peuvent être concernés. Les décès concernent environ $20 \%$ des cas et au moins $75 \%$ des bébés garderont des séquelles neurocognitives [2].

Il a été démontré que la majorité des cas de SBS surviennent sur des nourrissons âgés de 4 à 8 mois, ce qui correspond à la période où les bébés pleurent habituellement davantage [3].

Il n'existe pas de tableau clinique univoque et le diagnostic est difficile à évoquer quand les symptômes neurologiques ne sont pas au premier plan : vomissements parfois imputés à tort à une gastro entérite, anorexie, irritabilité ou pâleur anormale, arrêt cardio-respiratoire, etc. Les lésions squelettiques éventuellement associées sont souvent peu symptomatiques. L'auteur n'avoue jamais les faits de violence à l'hôpital et le radiologue est donc souvent le premier spécialiste à poser le diagnostic [4]. Rappelons que la moindre suspicion de maltraitance doit conduire à l'hospitalisation immédiate de l'enfant, puis à un signalement au Procureur de la République afin de garantir sa protection.

Il est nécessaire de corréler l'imagerie avec toutes les données cliniques, ophtalmologiques et biologiques. Certains éléments sont très évocateurs du syndrome du bébé secoué [2].

- une histoire clinique fluctuante ou incohérente,

- des ecchymoses surtout sur la face, les aisselles, le tronc ou le cou chez un nourrisson ne se déplaçant pas seul,

- une macrocrânie attestée par la courbe de périmètre crânien qui montre une brutale accélération de la vitesse de croissance,

- des hémorragies rétiniennes au fond d'œil, fortement corrélées au SBS quand elles atteignent la périphérie et/ou plusieurs couches de la rétine. Elles se résorbent rapidement et manquent dans environ $20 \%$ des cas [1].

\section{Quelle imagerie, quand et comment?}

Les recommandations des sociétés savantes du monde entier sont précises [1, 5-8]. Il faut effectuer chez tout enfant de moins de 2 ans suspect de maltraitance des radiographies de haute définition de l'ensemble du squelette, un scanner cérébral (au mieux complété par une IRM cérébrale) et il est recommandé en France d'effectuer largement une échographie abdominale à la recherche de lésions viscérales. La qualité des radiographies du squelette doit être extrêmement rigoureuse, segment par segment, car les fractures métaphysaires sont parfois très subtiles.

Une tomodensitométrie (TDM) cérébrale est systématique en phase aiguë et IRM largement conseillée en complément, dès que possible $[1,2]$. Les séquences axiales pondérées en $\mathrm{T} 1$, $\mathrm{T} 2, \mathrm{~T} 2 *$ et diffusion sont indispensables. La pondération $\mathrm{T} 2 *$ (écho de gradient) est très sensible à la détection de dépôts d'hémosidérine même de très petite taille (Fig. 1), grâce à l'artefact induit. Les séquences de susceptibilité magnétique (SWAN, SWI, venoBOLD), FLAIR axiales peuvent être utiles en complément. L'étude rachidienne peut aider en montrant un saignement intra-canalaire (Fig 1).

En l'absence de symptôme neurologique dans un contexte suspect de maltraitance, l'IRM cérébrale est recommandée en première intention à la recherche de lésions cicatricielles [5].

\section{L'imagerie, pourquoi ?}


Le signe caractéristique du syndrome du bébé secoué au scanner est la présence d'hématomes sous duraux très particuliers, multiples, interhémisphériques, sus et sous tentoriels. La prédominance de caillots à la convexité est un argument majeur indiquant une rupture des veines-ponts (Fig. 2) [1, 2, 8-10]. Les veines-ponts drainent le sang de la surface cérébrale vers le sinus longitudinal supérieur et leur point de fragilité se situe au niveau de l'espace sous dural. Ainsi, sous l'effet d'une accélération/décélération angulaire de la tête, les forces de cisaillement entrainent un étirement puis une rupture de ces veines ponts, responsable d'un hématome sous dural. La présence d'une rupture-thrombose de veines ponts est un indicateur très spécifique de traumatisme par secouement [11]. Le scanner permet parfois de visualiser une fracture du crâne et/ou une tuméfaction du scalp indiquant un impact associé aux secousses. Pour mémoire, le diagnostic d'une fracture du crâne est difficile en l'absence de «bosse » du fait de nombreuses variantes de la normale (sutures accessoires).

L'IRM permet souvent de visualiser directement les ruptures de veines ponts sous la forme de caillots tubulaires ou arrondis au sommet (Fig 3) et fournit des données complémentaires sur le parenchyme cérébral, notamment grâce aux séquences de diffusion en phase aiguë. Les lésions parenchymateuses sont souvent anoxo-ischémiques, de façon multifactorielle par compression par l'hématome sous dural, pauses respiratoires, état de mal épileptique, cascade excito toxique, stress oxydatif, etc. Les contusions sont souvent frontales et/ou temporales, du fait des impacts du cerveau contre les parois internes de la boite crânienne. Les lacérations frontales sont significativement associées à des traumatismes infligés (Fig. 4) [12].

L'IRM permet parfois de mettre en évidence un hématome rétroclival, des lésions traumatiques des muscles du cou, du canal rachidien et de la moelle épinière.

L'échographie transfontanellaire n'a pas de place dans le diagnostic de syndrome du bébé secoué car elle n'est pas sensible à la détection d'hématomes sous duraux.

Les radiographies du squelette recherchent des fractures subtiles, dont certaines sont directement liées au mécanisme du secouement, comme les fractures de côtes, témoignant de la compression thoracique violente de l'enfant ou les fractures-arrachements des métaphyses humérales proximales [13]. Les autres fractures-arrachements métaphysaires, fréquentes au niveau des genoux et des chevilles témoignent de maltraitance dans le cadre de torsions violentes des articulations, souvent provoquées lors des soins corporels à l'enfant [14]. Toutes les fractures peuvent se rencontrer [13].

\section{L'imagerie permet-elle de dater ?}

La datation appartient exclusivement à l'expertise médico-légale. Il est nécessaire de disposer de la totalité des auditions de l'enquête et des imageries effectuées depuis la naissance pour y répondre. Une datation effectuée à tort lors du diagnostic peut compliquer l'expertise médicolégale ultérieure.

Ni les ecchymoses ni les hémorragies rétiniennes ne peuvent être datées [2]. Aucune méthode d'imagerie ne permet de dater avec précision les lésions traumatiques, que ce soit au niveau cérébral ou du squelette. Dans le SBS, les hématomes sous duraux peuvent être hypo, iso ou hyperdenses et en pratique, ils présentent très souvent une densité hétérogène, «mixte » sans aucune signification en termes de datation : il peut s'agir de lésions aiguës ou hyper aiguës, de lésions d'âges différents, d'un mélange de sang et de liquide céphalo rachidien issu d'une brèche méningée (hémato-hygrome) ou d'une sédimentation. De plus, un hématome sous dural peut re-saigner spontanément du fait de membranes inflammatoires.

L'IRM apporte peu d'éléments supplémentaires pour dater les hématomes. La détection de membranes au sein d'un hématome sous dural est plus sensible qu'au scanner et indique un hématome d'au moins une huitaine de jours. Les anomalies en diffusion indiquent des lésions récentes ou semi récentes mais elles peuvent être liées à un état de mal convulsif secondaire au traumatisme. La présence d'une atrophie parenchymateuse, d'une cicatrice gliale localisée ou d'une porencéphalie indique des lésions anciennes datant de plus de 3 semaines à titre indicatif. Il est parfois possible de détecter des lésions traumatiques d'âges différents. Une 
étude basée sur les déclarations des auteurs a montré que les secousses, toujours très violentes et souvent déclenchées par les pleurs de l'enfant, sont répétées dans plus de la moitié des cas, parfois quotidiennement pendant plusieurs semaines [4]. Le secouement a pour effet immédiat de provoquer des troubles de conscience et donc de «calmer l'enfant», ce qui explique l'installation d'un cercle vicieux relationnel entre le nourrisson et l'auteur des violences. La répétition des lésions traumatiques explique également la difficulté de leur datation.

\section{L'imagerie et les diagnostics différentiels}

Le premier diagnostic à éliminer est le traumatisme accidentel. Seul un traumatisme avec forte décélération (accident de voiture) peut entrainer des hématomes sous duraux diffus avec ruptures de veines ponts observées dans moins de $10 \%$ des cas [11]. L'imagerie, combinée aux données cliniques et biologiques, permet également d'écarter les méningites, certaines maladies métaboliques, les anomalies de l'hémostase et les malformations vasculaires. Ces diagnostics ne sont pas associés à des ruptures de veines ponts. La macrocrânie banale du nourrisson est un diagnostic nécessitant l'analyse de la courbe de périmètre crânien depuis la naissance et ne s'accompagne d'aucun symptôme aigu ni de rupture de veines-ponts.

Concernant les fractures, le principal diagnostic différentiel est représenté par les maladies osseuses métaboliques et constitutionnelles mais les anomalies métaphysaires sont diffuses et stables dans le temps. L'ostéogenèse imparfaite (appelée aussi OI, maladie des os de verre, Lobstein, Porak et Durante) correspond à un groupe hétérogène de maladies génétiques caractérisées par une fragilité osseuse et une tendance aux fractures de sévérité variable mais il n'y a pas d'arrachements métaphysaires isolés et des os wormiens nombreux sont fréquents.

\section{En conclusion, qui explorer en imagerie ?}

La moindre suspicion de maltraitance doit conduire à une exploration exhaustive en imagerie (radiographies du squelette, imagerie cérébrale et échographie abdominale). Tous les enfants âgés de moins de 2 ans et ayant le même mode de garde (fratrie, nourrice...) que le cas index doivent être explorés de la même façon. Pour les enfants de plus de 2 ans, il n'y a pas de recommandation et, en pratique, le bilan complet est souvent effectué.

\section{RÉFÉRENCES}

[1] Haute Autorité de Santé (HAS). Syndrome du bébé secoué ou traumatisme crânien non accidentel par secouement. Recommandation de bonne pratique. Validation juillet 2017, mis en ligne le 29 septembr e2017. [En ligne] Disponible sur : https://www.hassante.fr/portail/jcms/c_2794425/fr/syndrome-du-bebe-secoue-ou-traumatisme-craniennon-accidentel-par-secouement (consulté le 12/07/2019)

[2]Rey-Salmon C, Adamsbaum C. Child abuse. Diagnostic and forensic considerations. Heidelberg: Springer, 2018, 434p.

[3] Barr RG. Crying as a trigger for abusive head trauma: a key to prevention. Pediatr Radiol. 2014;44(S4):S559-64.

[4]Adamsbaum C, Grabar S, Méjean N, et al. Abusive head trauma (AHT): judicial admissions highlight violent and repetitive shaking. Pediatrics 2010;126:546-55. Highlighted with editorial. Reece RM. Pediatrics. 2010;126:572-73.

[5] The American College of Radiology. ACR-SPR practice parameter for the performance and interpretation of skeletal surveys in children, revised 2016. [En ligne] Disponible sur : https://www.acr.org/-/media/ACR/Files/Practice-Parameters/SkeletalSurvey.pdf (consulté le 12/07/2019) 
[6]Royal College of Radiologists. Standards for Radiological Investigations of Suspected Non-accidental Injury. Joint document produced in collaboration with the Royal College of Pediatrics and Child Health. London: RCR, 2008. BFCR(08)1

[7] Société Francophone d'Imagerie Pédiatrique et Prénatale. [En ligne] Disponible sur : https://sfip-radiopediatrie.org/ (consulté le 12/07/2019)

[8]Choudhary AK, Servaes S, Slovis TL, et al. Consensus statement on abusive head trauma in infants and young children. Pediatr Radiol. 2018;48:1048-65.

[9]Rambaud C. Bridging veins and autopsy findings in abusive head trauma. Pediatr Radiol. 2015;45:1126-31.

[10] Ronning MM, Carolan PL, Cutler GJ, Patterson RJ. Parasagittal vertex clots on head CT in infants with subdural hemorrhage as a predictor for abusive head trauma. Pediatr Radiol. 2018;48:1915-23.

[11] Nahmani S. Rupture-thrombose des veines ponts dans les traumatismes crâniens accidentels et non accidentels: description scannographique et étude comparative. Thèse de médecine. Faculté de Médecine Paris Diderot, septembre 2018.

[12] Palifka LA, Frasier LD, Metzger RR, et al. Parenchymal brain laceration as a predictor of abusive head trauma. AJNR Am J Neuroradiol. 2016;37:163-8.

[13] Kleinman PK. Diagnostic imaging of child abuse. 3 ed. Cambridge: Cambridge University Press, 2015:750p.

[14] Adamsbaum C, de Boissieu P, Teglas JP, Rey-Salmon C. Classic Metaphyseal Lesions among victims of abuse. J Pediatr. 2019;209:154-159.e2 


\section{Légendes des figures}

Figure 1 : Enfant de 4 mois, syndrome du bébé secoué.

Coupe sagittale T2. Hématome intracanalaire dorsal en hyposignal (flèches courtes). Engagement des amygdales cérébelleuses (flèche longue)

Figure 2 : Scanner cérébral effectué chez un garçon âgé de 3mois pour vomissements.

Aspect typique de SBS. Hématome sous dural multifocal hypo et hyperdense (flèches). Caillots au sommet (image de droite, flèches).

Figure 3 : Rupture de veines-ponts. Syndrome du bébé secoué chez un nourrisson âgé de 3 mois.

Scanner (image à gauche) : doute sur une rupture-thrombose de veines-ponts (flèches noires). Effacement des sillons à droite.

IRM, coupe axiale T2*, sensible aux dépôts d'hémosidérine à 3 jours d'intervalle (image à droite) : confirmation de deux caillots tubulaires témoignant d'une rupture/thrombose de veines-ponts (flèches blanches) signant un mécanisme d'accélération-décélération.

Figure 4 : Lacérations frontales. IRM, coupe axiale T1 chez un bébé âgé de 2 mois.

Aspect très évocateur de traumatisme cérébral infligé. 

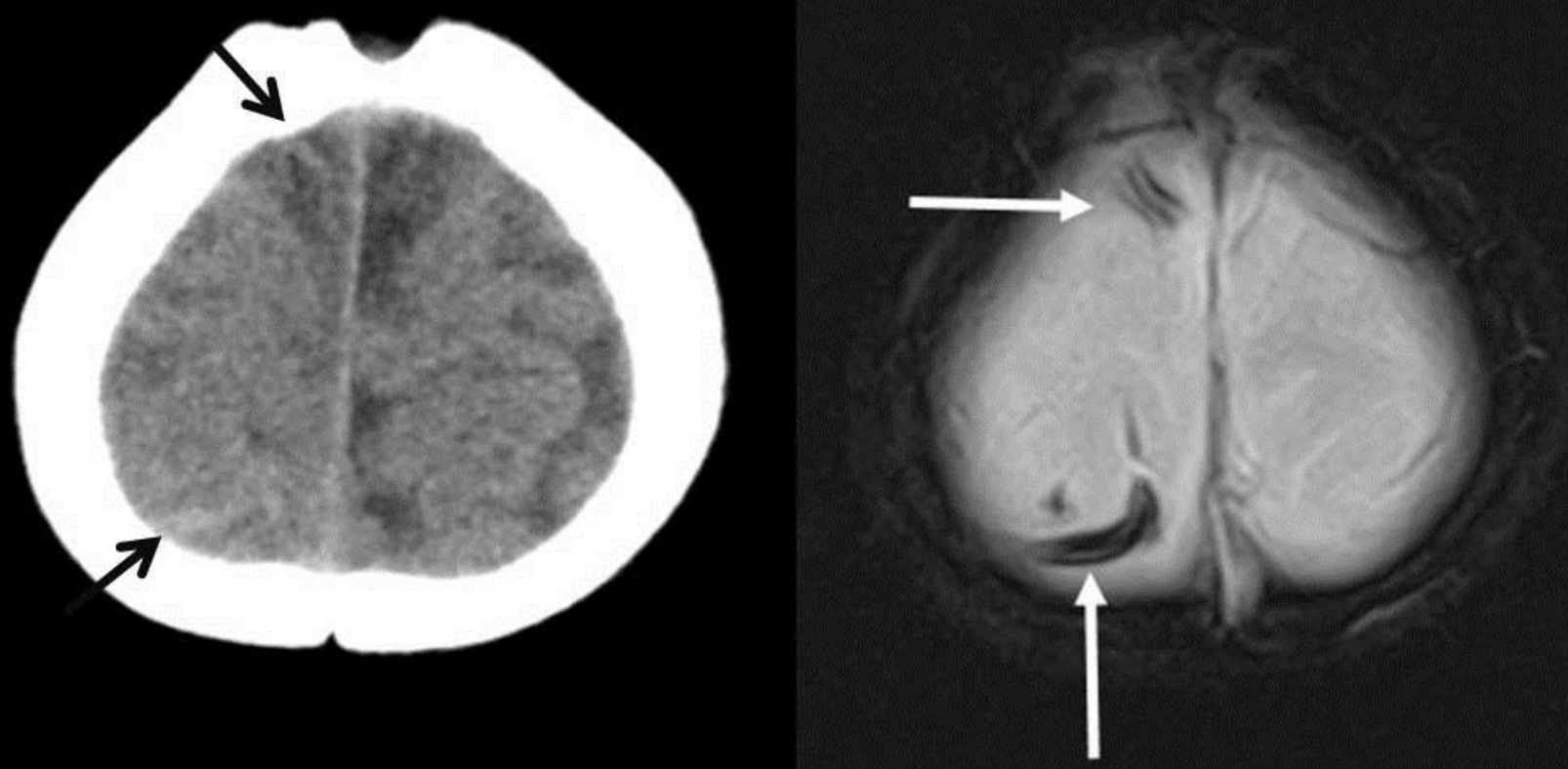


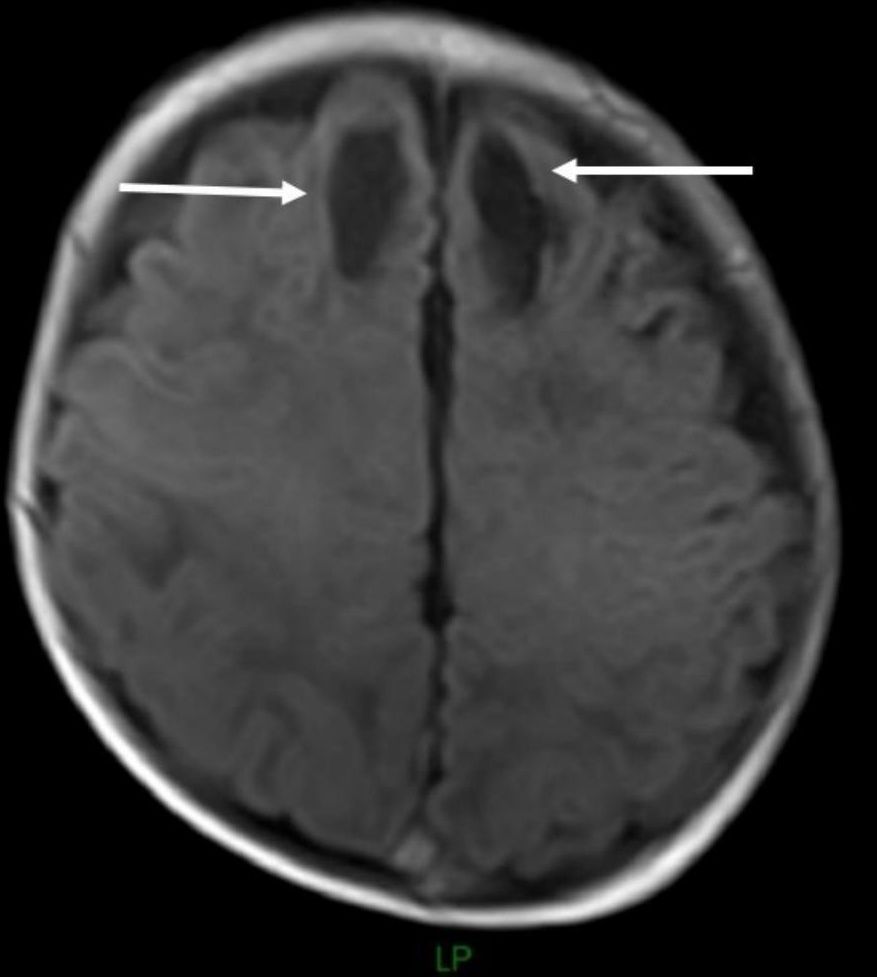

